\title{
Licensed Shared Access (LSA) trial demonstration using real LTE network
}

\author{
(Invited Paper)
}

\author{
Marko Palola ${ }^{1}$, Teemu Rautio ${ }^{1}$, Marja Matinmikko ${ }^{1}$, Jarmo Prokkola $^{1}$, Miia Mustonen ${ }^{1}$, Marjo Heikkilä ${ }^{2}$, Tero \\ Kippola $^{2}$, Seppo Yrjölä ${ }^{3}$, Vesa Hartikainen ${ }^{3}$, Lucia Tudose ${ }^{3}$, Arto Kivinen ${ }^{4}$, Jarkko Paavola ${ }^{4}$, Juha Okkonen ${ }^{5}$, Marko \\ Mäkeläinen ${ }^{5}$, Tuomo Hänninen ${ }^{5}$, and Heikki Kokkinen ${ }^{6}$
}

\begin{abstract}
This paper presents a demonstration of the new Licensed Shared Access (LSA) concept using a TD-LTE access network in the $2.3 \mathrm{GHz}$ spectrum band in Finland. In the demonstrated trial, the TD-LTE network shares the spectrum of an incumbent spectrum user, who is focused on programme making and special events (PMSE) services. The demonstrator implements the new LSA concept and the required new building blocks including LSA Repository for spectrum protection information and LSA Controller for controlling the mobile communication network in the same band. The trial uses commercial network components like multiband-terminals, TDLTE base stations, network management system, and core network. Spectrum usage data of incumbents is collected to LSA Repository using two incumbent user tools and is actively updated to LSA Controller for radio network planning to protect the incumbent's activity in the area. LSA Controller uses the information from the base stations and incumbents to protect incumbent spectrum users' rights. This is demonstrated by providing different algorithms, which analyze base stations and sectors that need to be reconfigured to prevent interference from LTE to incumbent (and vice versa). The demonstration shows that the new LSA concept can be implemented with existing network elements and a minimum amount of new components.
\end{abstract}

Keywords-field trial, live demonstration, spectrum sharing, mobile communication

\section{I.INTRODUCTION}

Mobile traffic is growing significantly towards 2020 [1]. This is leading to increasing spectrum demand for mobile network operators (MNOs). In addition to traditional exclusively licensed spectrum bands with long license durations, the availability of new exclusive bands that are restricted by the existing incumbent spectrum use, but are most of the time unused in large areas, should be considered as an opportunity in finding suitable frequency ranges for mobile communication systems.

A new spectrum sharing concept called the Licensed Shared Access (LSA) is a potential tool for introducing spectrum sharing between an MNO an another type of incumbent spectrum user. The LSA concept has received considerable interest in both European regulation and standardization for coordinating both the incumbent and MNO access in the same 2.3-2.4 GHz frequency band. As defined by

\footnotetext{
Marko Palola, Teemu Rautio, Marja Matinmikko, Jarmo Prokkola and Miia

Mustonen are with VTT Technical Research Centre of Finland, Oulu, Finland. firstname.lastname@vtt.fi

${ }^{2}$ Marjo Heikkilä and Tero Kippola are with Centria University of Applied Sciences, Ylivieska, Finland.

3 Seppo Yrjölä, Vesa Hartikainen and Lucia Tudose are with Nokia Solutions and Networks, Finland.

4 Arto Kivinen and Jarkko Paavola are with Turku University of Applied Criannos Turlu Finlond
}

the European Commission in [2], LSA is "a regulatory approach aiming to facilitate the introduction of radio communication systems operated by a limited number of licensees under an individual licensing regime in a frequency band already assigned or expected to be assigned to one or more incumbent users. Under the Licensed Shared Access (LSA) approach, the additional users are authorised to use the spectrum (or part of the spectrum) in accordance with sharing rules included in their rights of use of spectrum, thereby allowing all the authorized users, including incumbents, to provide a certain QoS".

The LSA concept [2] [3] allows spectrum sharing between an incumbent spectrum user and a licensee both having exclusive individual access to a portion of spectrum at a given location and time. The third key stakeholder in the LSA concept is spectrum regulator. The license sharing agreement between the incumbent and LSA licensee is implemented under the governance of the regulator. The regulator is responsible for defining the sharing framework and rules and conditions as well as issuing the license to the LSA licensee. The incumbent spectrum user defines the part of its spectrum that can be used for sharing with the LSA concept, the license duration and geographical area.

This paper focuses on demonstrating the LSA concept for sharing between an MNO and another type of incumbent spectrum user (e.g. PMSE in Finland). The LSA demonstration should not be mixed with previous works on spectrum sharing that have focused on unlicensed access to licensed spectrum bands like in the TV white space case [4]. In these approaches, the rights of the unlicensed users are not guaranteed from harmful interference, which is one of the key features of the LSA concept.

The LSA concept has been demonstrated for the first time in Finland in April 2013 [5] following updated features show in September 2013 [6] and April 2014 [7]. The demonstration of the LSA concept presented in this paper features more realistic LSA Repository interface with incumbent data notifications and evacuation confirmation messages as well as improved LTE network management operations allowing radio planning of individual sectors to protect the incumbent's business in the same area. 


\section{LSA TRIAL DEMONSTRATOR}

Demonstration of the LSA concept in the mobile network requires two new building blocks on top of the traditional cellular architecture, namely LSA Controller and LSA Repository, which are currently under consideration in standardisation [8] [9]. The key building blocks of the demonstrator are shown in Fig. 1 and include the following:

- Incumbent spectrum users and LSA Repository

- LTE network of three base stations with five sectors and end-user terminals

- LSA Controller including user interface for MNO and incumbent protection algorithms

- Visualization software.

The individual components and their interrelations are explained in more detail in the following sub-sections.

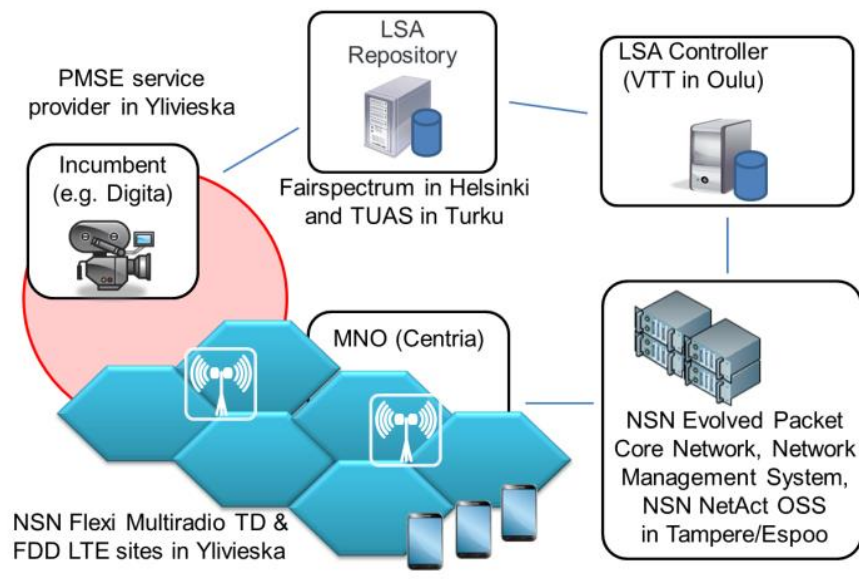

Fig. 1. Live trial environment for LSA.

\section{A. Incumbent spectrum users and LSA repository}

Incumbent spectrum users in this demonstration are assumed to be employees of a broadcasting company belonging to PMSE services, as defined in [10] and [11], and which utilizes parts of the 2.3-2.4 GHz band in Finland.

Fairspectrum LSA Repository and incumbent tools are developed by WISE2 project [12]. The LSA Repository is a database containing up-to-date information about LSA spectrum bands together with the conditions for the use of each band. LSA Repository could serve as an interface between the incumbent, the regulator and LSA Controller. LSA Repository collects, maintains and manages data on spectrum use and tracks whether LSA band is available for other purposes (as LTE in our case) or if it is in the incumbents' own use. LSA Repository contains information on organization, equipment, licenses, time, location, frequency, and type of the incumbent usage. Based on this information, protected areas are defined based on the underlying regulatory requirements. These protected areas are exclusion zones where the LSA users are not allowed to use the given LSA band.

Incumbent users are then requested to make a specific protection request to LSA Repository in order to notify the LSA users and possible other groups of incumbent users about the changes in frequency availability. The work load of the incumbent users should not increase and the information quality of the needed protection must be good. There are two tools for collecting incumbent reservations. The tools are LSA Incumbent Manager and PSME Location application. With

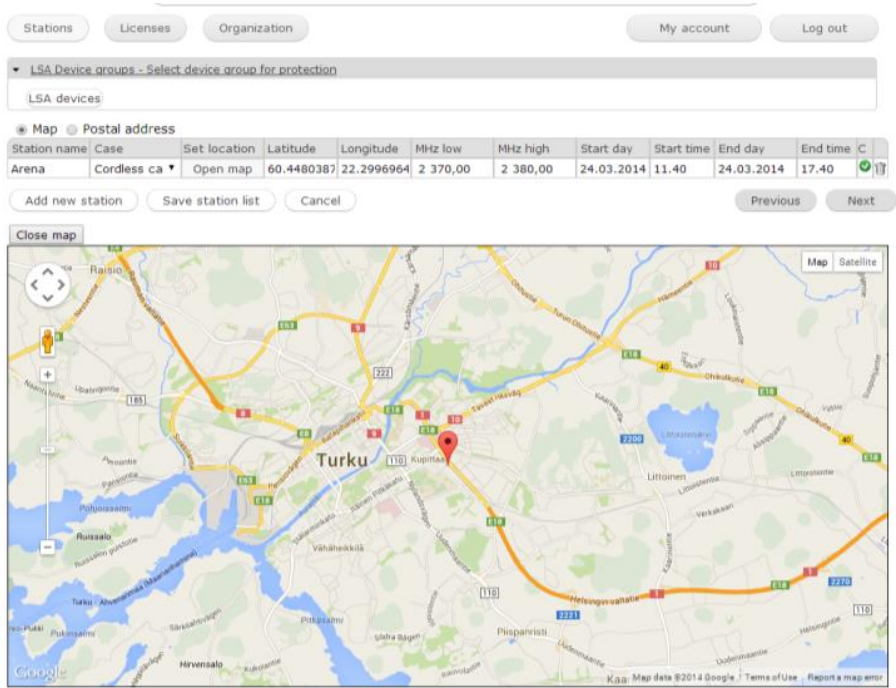

Fig. 2. LSA Incumbent Manager for the PMSE users.

these applications the incumbent user may set a transmit frequency range, request multiple protection areas, define a protection type and remove the protection from the LSA Repository.

The LSA Incumbent Manager tool (Fig. 2.) allows incumbent user to request multiple protections ahead, for example, to register next week's sport casting event. The incumbent user sets a location to the map interface, a protection type, a transmit frequency range and a casting time. The LSA Incumbent Manager processes the protection information and sends protection data to LSA Repository. The PMSE application (Fig. 3.) is connected to the LSA Incumbent Manager. The PMSE application uses mobile phone GPS to track the location of incumbent, which can be updated to LSA Repository accordingly.

The incumbent user can select between different protection types such as Cordless camera, a Mobile video link and a Portable video [10]. The protection type information includes information about a protection distance, transmit power limits and a reference to the broadcasting event setup. Different event setups include clear line-of-sight used with Cordless cameras, suburban area with no line-of-sight used with Portable video and cameras on-board vehicles used with Mobile video link. Protection type can be used in the MNO's protection algorithms to calculate more accurately protection area for each PMSE use.

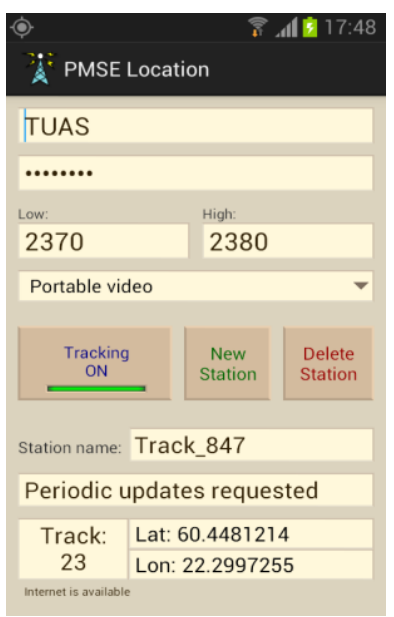

Fig. 3. PMSE Location application for incumbent users. 
LSA Repository contains the current incumbent's spectrum usage and it sends frequency reservation notifications to the MNO's LSA Controller when the information changes. In this demonstration, LSA Controller has the main responsibility of computing the protection criteria and implementing the terms of the sharing agreement between MNO and the incumbent. Based on the reservations and possible incumbent movement, MNO should act upon, and decide whether it can use LSA base station at certain location or not. The alternative implementation was demonstrated before, in which, LSA Repository was performing the required computations and giving directly the band availability for each base station.

\section{B. LTE network and terminals}

LTE network in the LSA demonstration consists of commercial 3GPP Release 8 compliant radio accesses and a real core network. Nokia Solutions and Networks (NSN) commercial LTE Advanced capable Flexi Multiradio 10 Base Stations are used. Three TD-LTE LSA base stations with five sectors located in Ylivieska, Finland, operate in the 2.36-2.40 $\mathrm{GHz}$ frequency band. Two FD-LTE base stations with four sectors that provide coverage to the same area in the $2.1 \mathrm{GHz}$ band, are for primary LTE use and are available also while LSA licensee is prohibited of using the LSA band.

The base stations are connected to LTE core network and are managed from a single point by the multi-technology, multi-vendor NetAct OSS platform. LSA Controller interfaces with the NetAct OSS and is able to get information about the LTE base stations and execute management operations to control them.

LTE multi-band (band 1 and 40) terminals are used. The TD-LTE implementation is rather new, but the iPhone $5 \mathrm{C} / \mathrm{S}$, and some of the latest USB dongles and Android smart phones support TD-LTE. In the demonstration we used Samsung S4 phones that also support dual-mode LTE providing TD-FDD seamless handover.

\section{LSA Controller and MNO UI}

The LSA Controller developed by the CORE+ project [13] provides the MNO with means to access the spectrum and to react on the incumbent user activity. In the demonstration, the MNO is able to receive de-activation and activation requests from the LSA Repository based on incumbent's frequency reservation reports. The incumbent's reservations include location, frequency bands, time range, and other information about the frequency use, for example, a type of cordless camera.

LSA controller also involves $\mathrm{MNO}$ user interface and incumbent protection algorithms. The MNO user interface can be seen in Fig. 4. It has three main sections: networks and users, LSA network features, and CORE+ environment status. The LSA controller gathers information on LTE base stations and sectors (both TD and FD) and their statuses through the network management system. The respective section in the figure depicts the existence of nine LTE sectors with their statuses. LSA spectrum band information and possible existence of incumbent(s) is also collected, and is presented in the same section of the UI. In the example, there is a single incumbent in an active mode and operating in the same band and area as the licensee, so LSA de-activation/evacuation process is currently ongoing.

The second section of the MNO UI is for controlling set of features with respect of LSA network, its usage, and

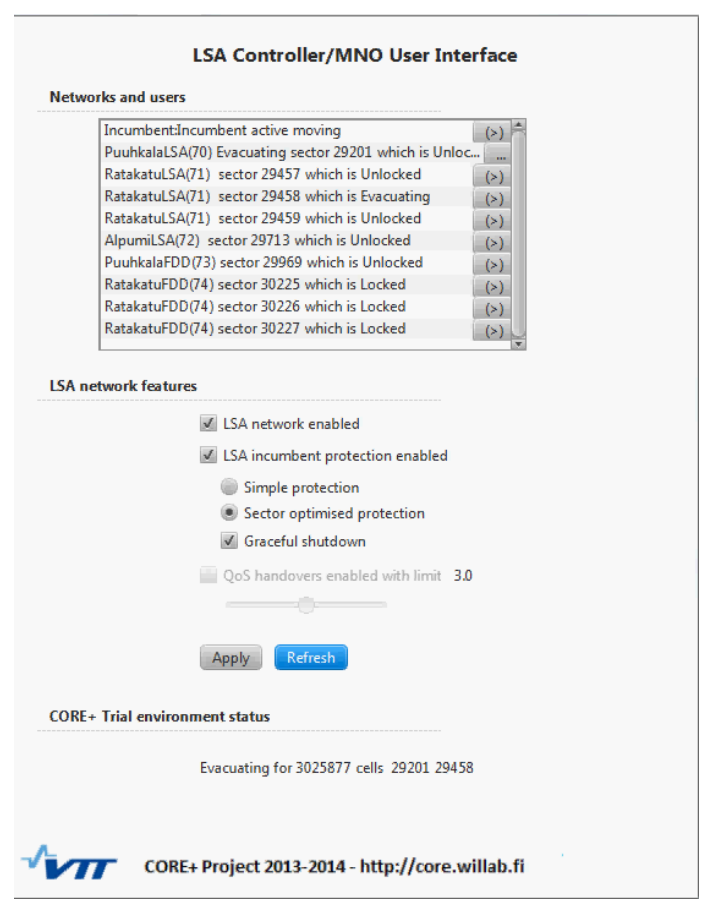

Fig. 4. Mobile network operators' user interface for selecting the demonstrator features and controlling the LSA network use.

incumbents' protection. With the first selection, called LSA network enabled, MNO can prevent the LSA controller of using the LSA base stations even though it could have possibility for using them. Similar manner, if LSA network is currently in use, and MNO unchecks this option, LSA controller will lock automatically the respective base stations.

With incumbent protection option, MNO can enable the band evacuation based on incumbent protection algorithms. MNO can choose between two protection algorithms. Simple protection calculates distance between the incumbent and base station and de-activates each base station within a certain radius. Sector optimized protection takes into account the sector angles and directions to select individual sectors for deactivation. Additionally, incumbent users located outside of a sector are protected if they are near to a base station site or a sector border. The demonstrator uses incumbent protection distances from [10] corresponding to the worst case scenarios.

The last option, a graceful shutdown, can be enabled to instead of shutting down a sector or base station immediately during de-activation, graceful shutdown lowers its power level step-by-step during the selected time period (35 seconds) before turning air interface off. This allows terminals to detect another network and carry out a seamless handover. Otherwise, the terminals have to go through a cell reselection, causing, potentially, a connection break to the ongoing session.

In practice, the real MNO's network is implemented as a Heterogeneous Networks (HetNet) topology consisting of various cell types like femtocells, picocells, microcells and macrocells and technologies like Wi-Fi, GSM, 3G and LTE each having different characteristics and base stations. The MNO is not willing to reveal this confidential information over the LSA Repository interface especially if the LSA Repository is located outside of MNO's network. Therefore, the algorithm in LSA Controller inputs the incumbent and base station information for MNO specific calculations. The algorithm outputs two lists. The first list contains sectors and incumbent users which experience interference and should be evacuated if sectors are active. The second list contains base 
stations and sectors that are not interfering with at least one of the incumbent users and are possible candidates for activation. However, a sector can be activated only if the same sector is not included to the other list (interfering with some other incumbent) and the sector is currently off air.

The QoS based handover selection is an additional feature of CORE+ system, allowing CORE+ monitored and controlled terminals to switch networks on demand. In this case, a QoS indicator can be used in a handover decision in addition to the base station signal strength. Measured QoS captures all quality decreasing effects, like network congestion, which the signal strength does not. In the trial, QoS information is provided with Qosmet [14].

\section{Visualization}

The demonstrator has graphical visualisation consisting of two parts. The first part is a topology view (Fig. 5.) for monitoring into which sector each LTE end-user terminals are at the time connected. For each terminal, the current data rate delivered over the air is represented. The topology view shows also Quality of Experience level indicator (indicated by the smileys in the figure), which is easily understood simple metric, but it is always an estimation, and works well only for certain applications. The second part of the visualisation is a map view (Fig. 6.). The LTE base stations (triangles in the figure), sectors of LTE network and sector states are depicted in the map view. The current LTE sector state is shown with different colours; green sector is active, red sector is being evacuated and grey sector has been evacuated. The map view also represents possible existence of the incumbent (diamond in the figure), in particular incumbent users' location and incumbents requested guard area.

\section{LSA DE-ACTIVATION ON INCUMBENT'S REQUEST}

The demonstrator features are

- Incumbent spectrum user's data and frequency requests are stored in the LSA Repository, which informs the LSA Controller about the changes in them and is able to update the incumbent location in real-time.

- LSA Controller manages TD-LTE base stations and individual sectors via the network management tool according to LSA band availability information.

- LSA Controller de-activates the base stations on LSA

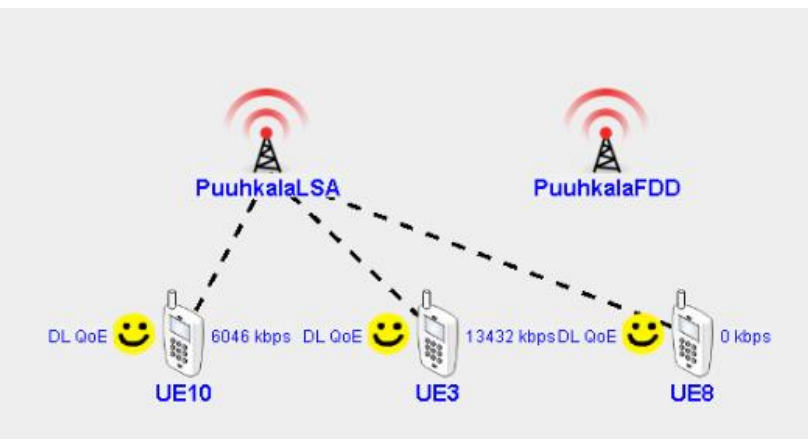

Fig. 5. Visualisation monitoring view for terminal streaming conditions and active connections

band on demand using either base station locking or graceful shutdown feature.

- The incumbent, licensee and regulator have defined and agreed the sharing framework including the rules and conditions for sharing. The regulator has granted a test license to use the $2.3 \mathrm{GHz}$ band for mobile broadband in the demonstrator area in Ylivieska, Finland.

The LSA demonstration focuses on the LSA concept's second phase, the LSA deployment [7] and features the following demonstration steps:

- LSA TD-LTE network is functional and the end user's terminals prefer LTE in the LSA band over the FDD band based on the signal strengths and handover limits set into the LTE network radio plan.

- Incumbent is able to define a frequency request at certain geographical are using the LSA Incumbent Manager tool. Evacuation request is stored in to the LSA Repository. The LSA Repository informs the LSA Controller about a new incumbent request.

- LSA Controller processes the incumbent and base station information, and selects base stations and sectors to be evacuated. LSA Controller commands the network management system to process a new radio network plan accordingly. The radio plan is provisioned to LTE network and selected sectors are turned off to evacuate the area and band for the incumbent.

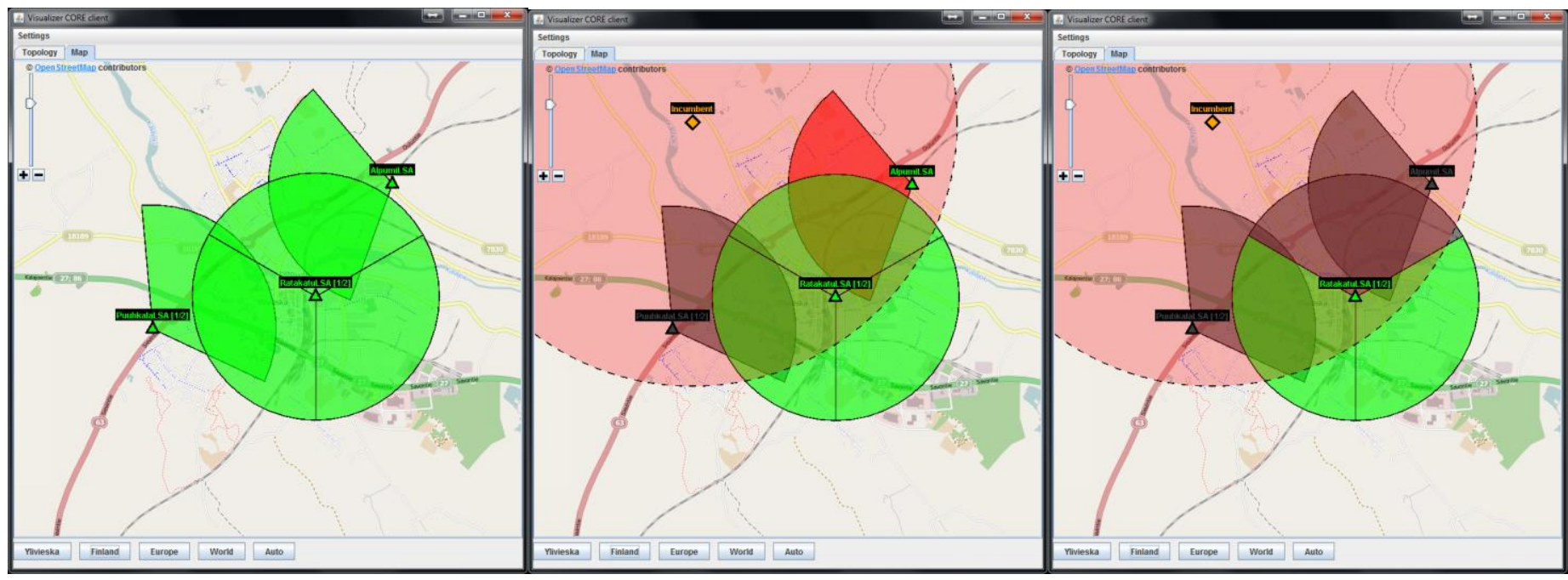

Fig. 6. Visualization view of the LTE LSA network with 5 sectors in different phases: without the incumbent (on the left), with the incumbent while the sector optimized evacuation process is in progress (in the middle) and with the incumbent after evacuation process has completed (on the right). 
- The terminals switch to another network. In demonstration the FDD-LTE is available as the main network coverage. Terminals carry out cell reselection in case the serving LSA sector is locked or a seamless handover if the LSA sector is gracefully shutdown by gradually lowering its power.

- The LSA Controller monitors the radio plan provisioning. As soon as the selected LSA sectors are setup accordingly the completion of the incumbent's band request is notified to the LSA Repository.

- The incumbent can safely begin using the LSA band as soon as the band reservation request is confirmed in the LSA incumbent management tool.

In the scenario, we are using several radio plans for each LSA base station (for locking and graceful shutdown of base station and individual sectors) and provisioning the plans one by one to the LTE network. Therefore, each sector evacuation using locking takes 20 seconds to complete and about 20-30 seconds more time when using 35 seconds period in graceful shutdown. By optimizing the radio plans, all the base stations and sectors can be managed using a single combined radio plan, making it possible to complete the evacuation within a couple of minutes in a relatively small area - if the LTE user experience is not managed. For specific incumbent needs, there is a fast evacuation support, which provides incumbent a possibility to request a large geographical area and band without specifying the intended use. In fast evacuation case, the sector and base station calculations are not used and the LSA band is temporary restricted from MNO use. Naturally, de-activating live base stations in a network may cause problems, at least, it activates LTE network load balancing and self-optimization routines [15] which could take hours before mobility and cell selections are again optimized in the adjacent cells.

\section{CONCLUSIONS}

This paper has presented a live demonstration of the new LSA concept for spectrum sharing between a mobile network operator (MNO) and another type of incumbent spectrum user. In particular, the trial demonstration has successfully shown that a TD-LTE network can take the shared $2.3 \mathrm{GHz}$ LSA band into use and evacuate it on a request by the incumbent spectrum user (PMSE in Finland). The demonstration showed that the dynamic availability of the LSA band can be handled with existing network equipment via the network management system extensions and a minimum number of additional components, namely a common LSA Repository and MNO specific LSA Controller.

LSA Repository can be kept relatively simple by hosting only incumbent protection data which is updated to the LSA Controller upon changes occurring in the incumbent band reservations and incumbent locations. Two tools are presented that ease the incumbent reporting workload by monitoring the incumbent location and providing a set of protection types for different PMSE use cases. LSA Controller can be hosting several types of incumbent protection algorithms which can take account the MNO specific data such as base station sites, power-levels, sectors and antennas. As soon as the incumbent data is received, the LSA Controller can calculate the needed protection area for each incumbent and select new radio plans accordingly which can be commissioned to the LTE network. Based on current conditions the LTE network configuration takes some time, so the confirmation of the incumbent band request is needed to update the LSA Repository and notify the incumbent about a safe band usage.

In the future, the LSA trials will be featuring more selforganizing networks functions. In particular, small cells, load balancing and traffic steering between different cell layers and radio access technologies will be interesting research topics. Interference measurements will be done in the LSA demonstration to help in defining the actual rules and conditions for sharing and incumbent protection. Key interest is to maximize the availability of the LSA spectrum while guaranteeing the incumbent's rights and LTE users.

\section{ACKNOWLEDGMENT}

This work has been done in the CORE+ and WISE2 research projects within the Trial program of Tekes - the Finnish Funding Agency for Technology and Innovation. The authors would like to acknowledge the CORE+ project consortium: VTT Technical Research Centre of Finland, University of Oulu, Centria University of Applied Sciences, Nokia Solutions and Networks, PehuTec, EXFO, Elektrobit, Anite, Rugged Tooling, Finnish Defence Forces, Finnish Communications Regulatory Authority, and Tekes. The authors would also acknowledge the WISE2 project consortium: Turku University of Applied Sciences, University of Turku, Aalto University, Fairspectrum, Nokia, Nokia Solutions and Networks, Teleste, Digita, Elektrobit, HSL, City of Jyväskylä, Finnish Communications Regulatory Authority, Satel, QEM Software, Viola Systems, and Tekes.

\section{REFERENCES}

[1] Report ITU-R M.2243. Assessment of the global mobile broadband deployments and forecasts for International Mobile Telecommunications. 2011

[2] RSPG Opinion on Licensed Shared Access, RSPG13-538, European Commission, Radio Spectrum Policy Group, November 2013.

[3] ECC Report 205. Licensed Shared Access. 2014

[4] CEPT, Technical and operational requirements for the possible operation of cognitive radio systems in the 'White Spaces' of the frequency band 470-790 MHz, ECC Report 159, Jan. 2011

[5] M. Matinmikko, M. Palola, H. Saarnisaari, M. Heikkila, J. Prokkola, T. Kippola, T. Hänninen, M. Jokinen, S. Yrjölä, "Cognitive Radio Trial Environment: First Live Authorized Shared Access-Based SpectrumSharing Demonstration, " IEEE Vehicular Technology Magazine, vol. 8, no. 3, Sept. 2013, pp. 30-37.

[6] LSA Trial Workshop. Helsinki, Finland, $3^{\text {rd }}$ of September 2013 [Online]. Available: http://tapahtumat.tekes.fi/event/triallsaworkshop.

[7] M. Palola, M. Matinmikko, J. Prokkola, M. Mustonen, M. Heikkilä, T. Kippola, S. Yrjölä, V. Hartikainen, L. Tudose, A. Kivinen, J. Paavola, and K. Heiska, " Live field trial of Licensed Shared Access (LSA) concept using LTE network in $2.3 \mathrm{GHz}$ band", in the 7th IEEE Symposium on New Frontiers in Dynamic Spectrum Access Networks (DySPAN), McLean, Virginia, USA, Apr. 1st-4th, 2014.

[8] ETSI. Mobile broadband services in the $2300 \mathrm{MHz}-2400 \mathrm{MHz}$ frequency band under Licensed Shared Access regime. ETSI System reference docyment. TR 103 113, V. 1.1.1. July 2013.

[9] J. Khun-Jush et al., "Licensed shared access as complementary approach to meet spectrum demands: Benefits for next generation cellular systems," in ETSI Workshop on Reconfigurable Radio Systems, Cannes, France, 2012.

[10] ECC Report 172. Broadband Wireless Systems Usage in 2300-2400 MHz. March 2012.

[11] ERC Report 38. Handbook on Radio Equipment and Systems Video Links for ENG/OB use. May 1995.

[12] WISE2 project web page [Online]. Available: http://wise.turkuamk.fi.

[13] CORE+ project web page [Online]. Available: http://core.willab.fi.

[14] J. Prokkola. Qosmet - Enabling passive QoS measurements [Online]. Available: http://www.cnl.fi/qosmet.html.

[15] S. Hamalainen, H. Sanneck and C. Sartori (Eds.); "LTE Self-Organizing Networks (SON)", John \& Wiley Sons, Ltd., 2012. 